\title{
Interdisciplinary Management of a Perforated Aneurysmal Arteria Lusoria: A Case Report
}

\author{
Mina Rouman ${ }^{1} \quad$ Alexander Petrovitch $^{2} \quad$ Eva-Maria Gey ${ }^{1}$ Thomas Kuntze ${ }^{1}$ \\ ${ }^{1}$ Deparment of Heart Surgery, Zentralklinik Bad Berka, \\ Bad Berka, Germany \\ 2 Deparment of Diagnostic and Interventional Radiology, Helios Klinik \\ Blankenhain, Germany \\ Address for correspondence Mina Rouman, Department of Heart \\ Surgery, Zentralklinik Bad Berka, Robert-Koch-Allee 9, Bad Berka \\ 99437, Germany (e-mail: mina_roman@yahoo.com).
}

Thorac Cardiovasc Surg Rep 2017;6:e15-e17.

\begin{abstract}
Keywords

- arteria lusoria

- aberrant right subclavian artery

- aneurysm

Background An aberrant right subclavian artery (RSA) or arteria lusoria is the most common congenital abnormality of the aortic arch with an incidence of 0.3 to $3.0 \%$. Case Description We report a case of a perforated aneurysmal aberrant RSA, managed using a hybrid approach.

Conclusion In emergency cases with acute bleeding, we recommend an endovascular approach to avoid the lethal sequel of arterial leakage. Whenever possible, the pulsatile blood flow to the right arm should be restored. Management should be tailored to the nature of the aneurysmal aberrant RSA, patient's comorbidities, and concomitant lesions.
\end{abstract}

\section{Introduction}

An aberrant right subclavian artery (RSA) or arteria lusoria is the most common congenital abnormality of the aortic arch with an incidence of 0.3 to $3.0 \%{ }^{1}$

The embryological origin of an aberrant RSA is the involution of the right fourth aortic arch, along with the cranial part of the right dorsal aorta, leaving the seventh intersegmental artery attached to the descending aorta. ${ }^{2}$ The aberrant RSA crosses between the esophagus and the vertebral column in $80 \%$ of cases. An aneurysmal aberrant RSA is a rare clinical entity. David Bayford ${ }^{3}$ was the first to use the term "dysphagia lusoria" to describe the clinical relevance of the aberrant RSA. He used the descriptive Latin term "dysphagia lusus naturae," that is, "freak of nature." An aberrant RSA is usually asymptomatic. ${ }^{4}$ However, it can manifest itself as dyspnea, chest pain, or dysphagia, and may clinically simulate pericarditis or aortic dissection. The management of the aberrant RSA' aneurysm is still debated. Different surgical and endovascular approaches have been used without a definite consensus. We report a case of a perforated aneurysmal aberrant RSA, managed using a hybrid approach.

\section{Case Description}

A 77-year-old man was admitted to the emergency department of a nearby hospital under suspicion of a stroke because of the sudden onset of left arm weakness. His chest X-ray showed a well-defined mass of the upper right mediastinum and an enlarged cardiac silhouette. A saccular aneurysm, $6 \mathrm{~cm}$ in diameter, was revealed in the computed tomography angiography (CTA). The patient was referred to our cardiovascular surgery center for further management.

The patient was hemodynamically stable at the time of admission. Laboratory tests revealed no abnormality. The patient was diabetic with a history of arterial hypertension, acute gastritis, and dysphasia to solid foods. Upon further questioning, he mentioned that he started having dysphagia 5 months ago, initially only to solid foods. After 2 to 3 months, he also noticed difficulty in swallowing soft foods.

He denied weight loss, nausea, vomiting, or chills. CTA showed a $6.2-\mathrm{cm}$ large aneurysm which was originating from the right lateral aspect of the aortic arch, reaching dorsal till the lumbar vertebrae where it passes behind the trachea (-Fig. 1). The spate arterial phase revealed a good perfused
License terms

Stuttgart · New York

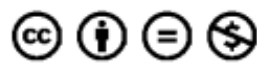

December 18, 2016 accepted after revision April 28, 2017
DOI https://doi.org/

10.1055/s-0037-1603625. ISSN 2194-7635. 


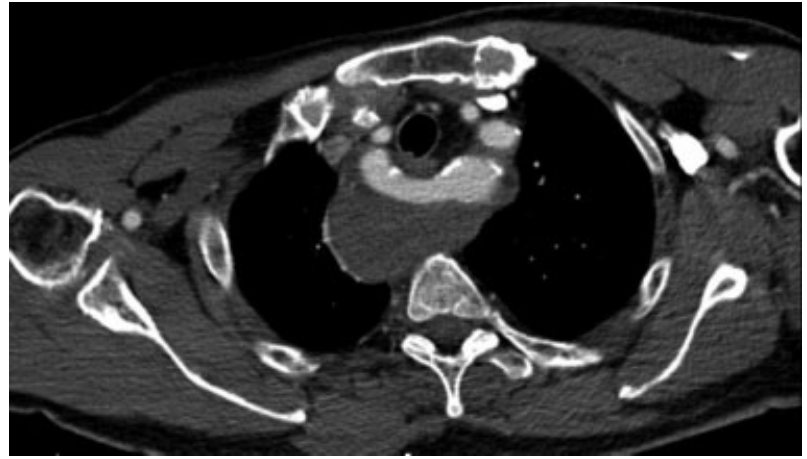

Fig. 1 CT axial plane at the level of the aneurysmal arteria lusoria course in superior mediastinum.

aneurysm with active bleeding and a coexisting truncus bicaroticus. There was no evidence of Kommerell's diverticulum or aneurysmal dilation of the aortic arch or root in the diagnostic angiography.

\section{Management}

As hybrid procedure, the patient underwent, in the first session, interventional closure of the arteria lusoria as follows: After surgical preparation of the right femoral artery, a $24 \mathrm{~F}$ femoral sheath was placed. Meanwhile a guiding catheter (Envoy MPC, 6F) was introduced in the right brachial artery. After closure of the aberrant RSA with a 24-mm Amplatzer plug2 (backdoor embolization), the perforation was coiled using 12 three-dimensional overlapping coils. Afterward, the aneurysmal sac was excluded from the

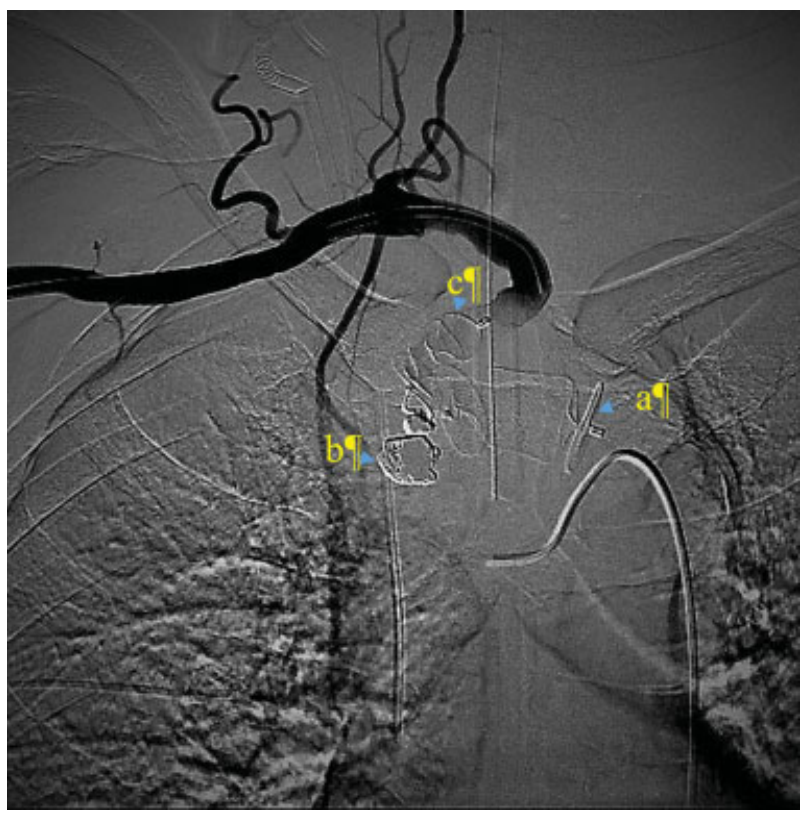

Fig. 2 (a) Closure of the arteria lusoria with a 24-mm Amplatzer plug2 (backdoor embolization); (b) coiled perforation using 3D overlapping coils; (c) right subclavian/arteria lusoria junction interrupted using a 16-mm Amplatzer plug2. remaining RSA using a 16-mm Amplatzer plug2 to avoid reversed perfusion of the aneurysmal sac with the subsequent steal phenomenon of the upper right limb and to avoid rupture of the perfused aneurysmal sac. Considering the coexisting truncus bicaroticus and the angiographic, free vertebral arteries, the estimated risk of a secondary stroke was minimal (-Fig. 2).

A right-sided carotid-subclavian bypass was not performed in the same operative session, after elimination of the aneurysm bleeding, due to the acceptable blood pressure difference between the two upper limbs ( $30 \mathrm{~mm} \mathrm{Hg}$ ) and to avoid the thoracotomy in this 77-year-old patient.

Nevertheless, after 24 hours, the patient developed signs of hypoperfusion of the right upper limb which was managed with a surgical transposition of the RSA to the right carotid artery.

Postoperatively, the patient was hemodynamically stable with no paresis. After the acute case management, a classical dysphagia workup was initiated. The patient's symptoms were relieved and postoperative CTA revealed no endoleak and the aneurysmal aberrant RSA was successfully excluded. The patient was discharged in good general condition. After 5 months, the patient felt subjectively good and performed his daily routine.

\section{Discussion}

Arteria lusoria results from abnormal embryologic development of the aortic arch. It is considered as a vascular ring.

The coexisting truncus bicaroticus was described from Klinkhamer ${ }^{5}$ as a prerequisite for tracheo-oesophageal compression and therefrom clinical symptoms. De Bakey et al in $1955^{6}$ described the first case of dissection of the descending aorta involving an aberrant RSA in a patient who also had truncus bicaroticus.

Gross $^{4}$ performed the first successful division and ligation of an aberrant RSA through a left thoracotomy. New endovascular techniques have been introduced successfully as a minimally invasive alternative in the management of aberrant RSA' aneurysm which might reduce the postoperative morbidity following thoracotomy. ${ }^{1}$

The surgical restoration of a pulsatile flow to the right arm is still controversial. ${ }^{4}$ Three patients of a series of six published by Kopp et $\mathrm{al}^{7}$ were treated by thoracic aortic stent graft and right-sided carotid-subclavian bypass. Severe upper-extremity ischemia or gangrene is rather a rare complication of a simple interventional exclusion of an aberrant RSA aneurysm. ${ }^{8}$ However, symptoms of hypoperfusion might not be eliminated. In case of this patient, postoperative underperfusion of the right arm was due to lack of anastomotic branches between the two subclavian arteries and also lack of collateral circulation around the shoulder.

In emergency cases with acute bleeding, we recommend an endovascular approach to avoid the lethal sequel of arterial leakage. Whenever possible, the pulsatile blood flow to the right arm should be restored. Management 
should be tailored to the nature of the aneurysmal aberrant RSA, patient's comorbidities, and concomitant lesions.

\section{References}

1 Kieffer E, Bahnini A, Koskas F. Aberrant subclavian artery: surgical treatment in thirty-three adult patients. J Vasc Surg 1994;19(01): 100-109, discussion 110-111

2 Janssen M, Baggen MG, Veen HF, et al. Dysphagia lusoria: clinical aspects, manometric findings, diagnosis, and therapy. Am J Gastroenterol 2000;95(06):1411-1416

3 Bayford D. An account of a singular case of obstructed deglutition. Mem Med Soc Lond 1794;2:275-286
4 Gross RE. Surgical treatment for dysphagia lusoria. Ann Surg 1946;124(03):532-534

5 Klinkhamer AC. Aberrant right subclavian artery. Clinical and roentgenologic aspects. Am J Roentgenol Radium Ther Nucl Med 1966;97(02):438-446

6 De Bakey ME, Cooley DA, Creech O Jr. Surgical considerations of dissecting aneurysm of the aorta. Ann Surg 1955;142(04):586-610, discussion, 611-612

7 Kopp R, Wizgall I, Kreuzer E, et al. Surgical and endovascular treatment of symptomatic aberrant right subclavian artery (arteria lusoria). Vascular 2007;15(02):84-91

8 Geiss D, Williams WG, Lindsay WK, Rowe RD. Upper extremity gangrene: a complication of subclavian artery division. Ann Thorac Surg 1980;30(05):487-489 